\title{
A Critical Review of Herbo-Mineral Formulations in Jwara Chikitsa
}

\author{
Akhila Retnam. $\mathrm{A}^{1}$, Keerthi V. $\mathbf{R}^{2}$, Reenu Mathew ${ }^{3}$, Sudheera. $\mathrm{S}^{4}$ \\ ${ }^{1,2} \mathrm{MD}$ (Ay), Ayurveda Physician, Self Employed, Kerala \\ ${ }^{3}$ Assistant Professor, Dept. of Koumarabhrithya, Govt. Ayurveda College, Thiruvananthapuram. \\ ${ }^{4}$ Associate Professor \& HOD, Dept. of Rasasastra \& Bhaishajya Kalpana, VPSV Ayurveda College, Kottakkal \\ Corresponding Author: Akhila Retnam. A
}

\begin{abstract}
Fever is a generalized body reaction to a variety of infectious and non-infectious causes. Fever was referred to as jwara by ancient Ayurvedic seers, and it was thought to be the most serious of all diseases. Fever is described as a separate illness, a symptom, and a complication of many ailments in Ayurveda classics. Jwara is the first and most serious of all diseases, so it needs special attention. In this article, we will attempt to compile and evaluate Rasa yogas in jwara chikithsa from various Ayurvedic classics. Rasendra Sara Sangraha, Bhaishjya Rathnavali, Rasa Ratna Samucchaya, Rasa Tharangini and other works are reviewed in detail. In Rasasastra classics, these formulations are indicated separately for navajwara chikitsa, nirama jwara chikitsa, jeerna jwara chikitsa, vishama jwara chikitsa, and sannipatha jwara chikitsa. This article includes 112 formulations from various sources that have been classified based on their indications. There are certain peculiarities in the ingredients based on jwara avasthas that can be discovered by studying these formulations. The majority of these formulations are herbo-mineral compounds that come in tablet form, making dosage, administration, and palatability more convenient. A few of these formulations are commercially available for common clinical practise. Antipyretic use is excessive in everyday life in the present pandemic situation. The aim of this study is to raise awareness of these particular formulations in the health-care system so that they can be applied to community needs.
\end{abstract}

Key words: fever, jwara, navajwara, vishama jwara, nirama jwara, sannipatha jwara, herbo mineral formulations, rasa yogas

\section{INTRODUCTION}

Fever has been a characteristic of the disorder since the beginning of recorded history. Fever is the most popular excuse for a visit to the doctor these days. It is the body's complicated response to a variety of immune threats. It can also be used to predict current or potential medical problems. Jwara is not just a vyadhy, according to Ayurveda. It is a Lakshana as well as an upadrava for a variety of ailments. As a result, it is critical from a management perspective. The set of Rasayogas listed in jwara chikithsa prakaranas of various classical textbooks is one of the main goals of the study. We can see that these yogas are appropriate for various jwara avasthas, such as Nava, Nirama, Jeerna, Vishama, and Sannipatha, by examining them. Hence, they have been grouped together based on their indications. With a detailed examination we can found that each group contains nearly identical herbal and mineral ingredients. Mineral drug ratios, bhavana dravyas, dosage and anupana, on the other hand, differ. There are also some ingredients in each category that are unusually noticeable. In nava jwara hara yogas, for example, visha and upavisha dravyas are used as a common 
ingredient or bhavana dravya. Such ingredients are uncommon in nirama jwara hara yogas.

Rasa yogas in Ayurveda have a wide range of therapeutic utility and are fastacting even in small doses. With proper anupana, herbo mineral formulations can be recommended more safely and effectively in a variety of pathological conditions. Most of the herbo mineral formulations described in Rasasastra classics are tablet form in jwara chikitsa, making their dosage, mode of administration, and palatability, among other things, more convenient.

\section{Background and rationale}

According to Acharya Charaka, Jwara is the first and most serious of all diseases, so it needs special attention. Fever is described as a separate illness, a symptom, and a complication of many ailments in Ayurveda classics. Despite the advancement of contemporary science, fever remains the first and foremost symptom of almost all clinical consultations.

"While the focus is on the rising number of COVID-19 cases in Kerala, the fact is that a sizeable number of people die in the state due to fever. According to official statistics, the state has recorded 234 fever deaths in the past five years, including 51 last year." "In 2018 and 2017, 63 and 76 fever deaths were reported, respectively, in the state. Experts say that the number of deaths due to fever will be much higher and "scary" than the official figures as most fever deaths go unreported." "Fever is the most dangerous because it does not reveal the disease; it is only a symptom that can lead to something major at times. Fever is a symptom of over 1,000 diseases and, therefore, it is very important that the fever is kept in check if one gets it. If we look at the fever death cases in all age groups, from babies to elderly, the number would be a scary one. The fever death cases in private hospitals are not included in the official statistics, so the real number would be twice the official figure," said Dr Anup R Warrier, infectious diseases expert." 1

This is according to the current statistics from Kerala, where even in rural regions, well-established medical services, including doctors and laboratory facilities, are available. When we examine India as a whole, as well as the rest of the world, the situation is dire. The purpose of this work is to familiarize and remember the potential Ayurveda medicines in light of the current pandemic crisis.

\section{Jwara Samprapthi \& Types in Ayurveda classics}

Acharya Charaka gave various names to Jwara in Ayurveda, including 'Vikara', Roga', 'Vvyadhi', 'Aathanka' and so on ${ }^{2}$. Fever is caused by deranged doshas of the body and mind, as it does not occur in people who are free of them ${ }^{3}$. Pyrexia (santhapa), anorexia (aruchi), thirst (trishna), body ache (anga marda), and distemper are some of the symptoms associated with fever ${ }^{4}$. When the increased doshas enter Amasaya and combine with undigested or improperly processed metabolites (aama) present in the alimentary canal (amasaya), fever is produced. These doshas with ama then block the channels of the rasa dhathu, removing the digestive fire from its usual location (pakthi sthana) ${ }^{5}$. Increased body temperature is the cardinal characteristic of fever, as the digestive fire pushes to the skin and all other body tissues. Sweating does not occur in general, despite the rise in temperature, due to the doshas' and ama's blockage of the sweat channels ${ }^{6}$.

When it comes to jwara categorization, different textbooks have different categories such as ashta vidha, pancha vidha, dvi vidha and so on ${ }^{7}$. The pharmaceutical formulations are listed in this article according to the stages of fever, as in the Rasendra sara sangraha. Hence, the jwara is examined in terms of its stages. 


\subsection{Nava jwara in Ayurveda}

According to the knowledgeable academics of Ayurveda, the Tharuna jwara refers to the period from the first to the seventh day. The intermediate stages (Madhyama jwara) of the fever correspond to the period from the seventh to the twelfth night. If the fever lasts longer than that, it is considered Purana jwara ${ }^{8}$.

The jwara samprapthi is influenced by the development of ama. Except for vathika and kshayaja conditions, ama soshana and ama nirharana are the key aspects of Nava jwara chikithsa. Drugs with kashaya rasa (astringent taste) and decoctions (kwatha preparations) are not recommended in nava jwaravastha ${ }^{9}$. If we take decoctions at that time, the fever would worsen, and the vitiated dosha will be exceedingly difficult to manage ${ }^{10}$. Langhana, swedana, kala, and yavagu prepa red with thiktha rasa dravyas are general tre atment modalities for tharuna jwara $^{11}$. In the case of an acute fever, the pa tient should avoid day time sleep, bathing, anointing, heavy food, sexual activity, wrath , wind, exercise, and astringent decoctions ${ }^{12}$.

\subsection{Nirama jwara}

The signs and symptoms of nirama jwara include an increase in appetite, a lighter body, a decrease in temperature, and the evacuation of doshas as well as waste materials from the body. On the eighth day of a fever, these signs and symptoms usually occur. $^{13}$

\subsection{Sannipatha jwara}

The treatment principles of sannipatha jwara are langhana, valuka sweda (fomentation with hot sand), nasya (inhalation therapy), nishteevana (spitting therapy), avaleha or confection administration, and anjana therapy ${ }^{14}$. The vitiated ama kapha should also be treated first with langhana, swedana, and nasya therapies in sannipatha jwara ${ }^{15}$. The procedures for pacifying pitta and vatha should be undertaken after achieving kapha ksheenavastha.

\subsection{Vishama jwara}

Vitiation of all doshas (sannipatha) leads to the vishama jwara. Therefore, the dosha with the maximum vitiation should be treated first ${ }^{16}$. Measures such as vamana and virechana therapies are also advised in the treatment of this form of fever. Fever can also be lowered by eating and drinking greasy, hot foods and drinks (snigdha \& ushna anna pana).

\subsection{Jeerna jwara}

This indisposition is called jeerna jwara or chronic fever if the degree of fever is decreased after 21 days and only affects certain parts of the body such as the hands, feet, and shoulders. Symptoms include spleen enlargement (pleeha) and loss of digestive ability (agni saadam) ${ }^{17}$. The patient should be treated with peya, kashaya,sarpi and ksheera,on every sixth day purgation therapy should be administered according to the bala of patient 18 .

\section{MATERIALS AND METHODS}

The principal sources for this study were key Ayurvedic books, ranging from Charaka Samhitha to Rasarathna Samuchaya, Rasa Tarantini, and Rasendra Sara sangraha. Rasendra Sara sangraha is particularly helpful for this article, which categorizes rasa yogas based on jwara avasthas ${ }^{19}$ and refers to other works for the other essential formulations. This work only includes the herbo-mineral formulations mentioned for jwara chikithsa. Attempt was made to analyze them based on their ingredients, such as minerals, herbs, and special drug groups such as upa visha varga, pancha pitha, and so on. 


\section{List of herbo mineral formulations with their ingredients and bhavana dravyas}

Chart 5.1- Herbo mineral formulations in nava jwara

\begin{tabular}{|c|c|c|c|c|c|c|}
\hline $\begin{array}{ll}\text { SI. } \\
\text { NO }\end{array}$ & Name of book & Name of yoga & $\begin{array}{l}\text { Mineral } \\
\text { ingredients }\end{array}$ & Herbal ingredients & $\begin{array}{l}\text { Visha/ } \\
\text { upaviha }\end{array}$ & Bhavana dravya \\
\hline 1 & $\begin{array}{l}\text { BR, Jwara } \\
\text { chi.,528-533 }\end{array}$ & Agni kumara rasa & & Maricha, vacha, musthaka & Visha & Ardraka rasa \\
\hline 2 & $\begin{array}{l}\text { RSS Jwara. Chi., } \\
\text { sloka- } 44,45\end{array}$ & $\begin{array}{l}\text { Amrutha manjari } \\
\text { rasa }\end{array}$ & Hingula, tankana & Maricha, pippali, jathikosa & Visha & Jambeeraswarasa \\
\hline 3 & $\begin{array}{l}\text { BR, Jwara chi.- } \\
575-584\end{array}$ & $\begin{array}{l}\text { Achinthyasakthi } \\
\text { rasa }\end{array}$ & $\begin{array}{l}\text { Rasa, gandhaka, } \\
\text { swarnamakshika }\end{array}$ & maricha & & $\begin{array}{l}\text { Bringakesa, } \\
\text { manduki ,patra } \\
\text { etc. }(9 \text { drugs) }\end{array}$ \\
\hline 4 & $\begin{array}{l}\text { BR (Jwara. Chi., } \\
\text { sloka-484) }\end{array}$ & $\begin{array}{l}\text { Brihath } \\
\text { hinguleswara rasa }\end{array}$ & Hingula, tankana & Vyosha, nagara & $\begin{array}{l}\text { Visha, } \\
\text { Jaipala }\end{array}$ & \\
\hline 5 & $\begin{array}{l}\text { BR,Jwara. Chi., } \\
\text { sloka-569-572 }\end{array}$ & Chandeswara rasa & $\begin{array}{l}\text { Rasa, gandhaka, } \\
\text { Tamra }\end{array}$ & & Visha & $\begin{array}{l}\text { Ardraka rasa, } \\
\text { nirgundi swarasa }\end{array}$ \\
\hline 6 & $\begin{array}{l}\text { RSS Jwara. Chi., } \\
\text { sloka-41 }\end{array}$ & Gadamurari rasa & $\begin{array}{l}\text { Hg,Gandaka,Sila, } \\
\text { Loha, Tamra, } \\
\text { Darada,Naga }\end{array}$ & Vyosha & & Water \\
\hline 7 & $\begin{array}{l}\text { RSS Jwara. Chi., } \\
\text { sloka-2 }\end{array}$ & Hinguleswara rasa & Hingula & Pippali & Visha & \\
\hline 8 & $\begin{array}{l}\text { RSS Jwara. Chi., } \\
\text { sloka-16 }\end{array}$ & Jaya vati & & $\begin{array}{ll}\begin{array}{l}\text { Trikatu, mustha, } \\
\text { nimbapatra,vidanga }\end{array} & \text { haridra, } \\
\end{array}$ & $\begin{array}{ll}\text { Jaya } & -8 \\
\text { part } & \\
\end{array}$ & Chagamutra \\
\hline 9 & $\begin{array}{l}\text { RSS Jwara. Chi., } \\
\text { sloka-17 }\end{array}$ & Jayanthi vati & & Pata, aswagandha, vacha etc. & $\begin{array}{l}\text { Visha, } \\
\text { jayanthi }\end{array}$ & Ajamuthra \\
\hline 10 & $\begin{array}{l}\text { RSS Jwara. Chi., } \\
\text { sloka-3 }\end{array}$ & $\begin{array}{l}\text { Jwara dhumakethu } \\
\text { rasa }\end{array}$ & $\begin{array}{l}\mathrm{Hg}, \mathrm{S} \text {,hingula, } \\
\text { samudraphena }\end{array}$ & & & Ardrakaswarasa \\
\hline 11 & $\begin{array}{l}\text { RSS Jwara. Chi., } \\
\text { sloka-35 }\end{array}$ & Jwaramurari rasa & Hingula, tankana & Vyosha, nagara, abhaya & $\begin{array}{l}\text { Visha, } \\
\text { jayapala }\end{array}$ & \\
\hline 12 & $\begin{array}{ll}\text { BR,Jwara } & \text { chi. } \\
560-561 & \\
\end{array}$ & Jwarahari rasa & $\begin{array}{l}\text { Seesaka, Rasa } \\
\text { sindura, thala }\end{array}$ & & Visha & Water \\
\hline 13 & $\begin{array}{l}\text { BR, Jwara chi. } \\
585-590\end{array}$ & Jwarakesari rasa & Rasa, gandhak & Vyosha, triphala & $\begin{array}{l}\text { Jaypala, } \\
\text { Visha }\end{array}$ & Bhringatoya \\
\hline 14 & $\begin{array}{l}\text { RSS Jwara. Chi., } \\
\text { sloka- }\end{array}$ & Jwarasimha rasa & $\begin{array}{l}\text { Parada, } \\
\text { Gandhaka, Tala }\end{array}$ & & $\begin{array}{l}\text { Bhallatha } \\
\text { ka }\end{array}$ & Vajriksheera \\
\hline 15 & $\begin{array}{l}\text { RSS Jwara. Chi., } \\
\text { sloka (4-15) }\end{array}$ & Mrithyunjaya rasa & $\begin{array}{l}\text { Gandaka hingula, } \\
\text { tankana }\end{array}$ & Pippali, maricha & Visha & \\
\hline 16 & $\begin{array}{l}\text { RSS Jwara. Chi., } \\
\text { sloka-1 }\end{array}$ & $\begin{array}{l}\text { Nava jwarankusa } \\
\text { rasa }\end{array}$ & $\begin{array}{l}\mathrm{Hg}, \quad \mathrm{S}, \quad \text { Hingula } \\
(1: 2: 3)\end{array}$ & & \begin{tabular}{l}
\multicolumn{2}{l}{ Danthibee } \\
ja $\quad(4$ \\
parts)
\end{tabular} & $\begin{array}{ll}\text { Danthi moola } \\
\text { kwatha }\end{array}$ \\
\hline 17 & $\begin{array}{l}\text { BR, Jwara chi- } \\
522\end{array}$ & $\begin{array}{ll}\text { Nava } & \text { jwarankusa } \\
\text { rasa } & \\
\end{array}$ & Rasa, S, hingula & & $\begin{array}{l}\text { Nikumbha } \\
\text { beeja }\end{array}$ & Danthi varina \\
\hline 18 & $\begin{array}{l}\text { RSS (Jwara. } \\
\text { Chi., sloka- 36) }\end{array}$ & $\begin{array}{l}\text { Nava } \\
\text { jwarebhankusa rasa }\end{array}$ & $\begin{array}{l}\text { Hg, S, Talaka, } \\
\text { Tankana }\end{array}$ & & & Meenapitha \\
\hline 19 & $\begin{array}{l}\text { RSS Jwara. Chi., } \\
\text { sloka-55-57 }\end{array}$ & $\begin{array}{l}\text { Nava jwarebha } \\
\text { simha rasa }\end{array}$ & $\begin{array}{l}\mathrm{Hg}, \quad \mathrm{S}, \quad \text { loha, } \\
\text { tamra, seesa }\end{array}$ & Maricha, pippali, viswa & Visha & \\
\hline 20 & $\begin{array}{l}\text { BR, Jwara chi. } \\
498-499\end{array}$ & Nava jwarahari vati & $\mathrm{Hg}, \mathrm{S}$ & Trikatu, triphala & $\begin{array}{l}\text { Danthibija } \\
\text {, visha }\end{array}$ & Dronapushpi rasa \\
\hline 21 & $\begin{array}{l}\text { BR, Jwara } \\
\text { chi.500-502 }\end{array}$ & Nava jwarari rasa & Rasa, gandhaka & Swarnaksheeri & $\begin{array}{l}\text { Visha(3pa } \\
\text { rts),jayapa } \\
\text { la(5 parts) } \\
\end{array}$ & Nimbu rasa \\
\hline 22 & $\begin{array}{l}\text { BR,Jwara } \\
\text { chi.523-524 }\end{array}$ & $\begin{array}{l}\text { Prachandeswara } \\
\text { rasa }\end{array}$ & Rasa, gandhaka & & Visha & Sindhuvara rasa \\
\hline 23 & $\begin{array}{l}\text { BR, Jwara. Chi., } \\
\text { sloka-839-858 }\end{array}$ & $\begin{array}{l}\text { Prathapalankeswara } \\
\text { rasa }\end{array}$ & Hingula, tankana & & $\begin{array}{l}\text { Visha, } \\
\text { jayapala }\end{array}$ & \\
\hline 24 & $\begin{array}{l}\text { RSS Jwara. Chi., } \\
\text { sloka-38 }\end{array}$ & $\begin{array}{l}\text { Prathapamarthanda } \\
\text { rasa }\end{array}$ & Hingula, tankana & & $\begin{array}{l}\text { Jaypala, } \\
\text { visha }\end{array}$ & \\
\hline 25 & $\begin{array}{l}\text { BR,Jwara chi. } \\
562-567\end{array}$ & Ratnagiri rasa & $\begin{array}{lll}\mathrm{Hg}, & \mathrm{S}, & \text { tamra, } \\
\text { loha, } & \text { abhra, } \\
\text { swarna } & \\
\end{array}$ & $\begin{array}{l}\text { Sigru, vasa, nirgundi, } \\
\text { vacha,chitraka, bhringa raja etc. }\end{array}$ & & \\
\hline 26 & $\begin{array}{l}\text { BR,Jwara. Chi., } \\
\text { sloka-638-641 }\end{array}$ & $\begin{array}{l}\text { Sannipatha bhairava } \\
\text { rasa }\end{array}$ & Tamra,S, Hg & Harithaki, maricha & $\begin{array}{l}\text { Swethagu } \\
\text { nja, } \\
\text { jayapala }\end{array}$ & Meenapitha \\
\hline 27 & $\begin{array}{l}\text { BR, Jwara } \\
\text { chi,503-505 }\end{array}$ & $\begin{array}{l}\text { Sarvangasundara } \\
\text { rasa }\end{array}$ & $\begin{array}{l}\text { Rasa } \\
\text { gndhakatankana }\end{array}$ & Triphalatrikatu & $\begin{array}{l}\text { Visha, } \\
\text { jayapala }\end{array}$ & \\
\hline 28 & $\begin{array}{l}\text { BR,Jwara. Chi., } \\
\text { sloka-485-487 }\end{array}$ & Seethabhanji rasa & $\mathrm{Hg}, \mathrm{S}$, hingula & & Jaypala & Danthikwatha \\
\hline 29 & $\begin{array}{l}\text { BR, Jwara. Chi., } \\
\text { sloka-520-521 }\end{array}$ & Sri rama rasa & $\mathrm{Hg}, \mathrm{S}$ & Maricha (2parts) & $\begin{array}{l}\text { Jaipalabee } \\
\text { ja }\end{array}$ & Danthikwatha \\
\hline 30 & $\begin{array}{l}\text { BR,Jwara. Chi., } \\
\text { sloka-490-491 }\end{array}$ & $\begin{array}{l}\text { Swachandabhairva } \\
\text { rasa }\end{array}$ & Tamrabhasma & & $\begin{array}{l}\text { Visha } \\
\text { hema }\end{array}$ & Dhathuraswarasa \\
\hline 31 & $\begin{array}{l}\text { BR Jwara. Chi., } \\
\text { sloka-488-489 }\end{array}$ & Tarunajwaraari rasa & $\mathrm{Hg}, \mathrm{S}$ & & $\begin{array}{l}\text { Visha, } \\
\text { jaipala }\end{array}$ & Kumari rasa \\
\hline
\end{tabular}


Akhila Retnam. A et.al. A critical review of herbo-mineral formulations in jwara chikitsa.

\begin{tabular}{|c|c|c|c|c|c|c|}
\hline \multicolumn{7}{|c|}{ Chart 5.1 Continued.... } \\
\hline 32 & $\begin{array}{l}\text { BR,Jwara. Chi., } \\
\text { sloka-558 }\end{array}$ & $\begin{array}{l}\text { Trilokyatambara } \\
\text { rasa }\end{array}$ & $\begin{array}{ll}\mathrm{Hg}, & \mathrm{Cu}, \mathrm{S}, \\
\text { Chapala } & \end{array}$ & Tiktha, pathya, trivruth & $\begin{array}{l}\text { Jaipala } \\
\text { vishathint } \\
\text { huka }\end{array}$ & Vajripayas, madhu \\
\hline 33 & $\begin{array}{l}\text { BR,Jwara. Chi., } \\
\text { sloka-506-508 }\end{array}$ & $\begin{array}{l}\text { Triputrabhairava } \\
\text { rasa }\end{array}$ & $\begin{array}{ll}\begin{array}{l}\text { Tamra, } \\
\text { tankana }\end{array} & \mathrm{S}, \\
\end{array}$ & & Danthibija & $\begin{array}{l}\text { Danthimoolakwat } \\
\text { ha }\end{array}$ \\
\hline 34 & $\begin{array}{l}\text { BR,Jwara, 573- } \\
574\end{array}$ & Udakamanjari rasa & $\mathrm{Hg}, \mathrm{S}$, tankana & Maricha & & $\begin{array}{l}\text { Sitha, mathsya } \\
\text { pitta }\end{array}$ \\
\hline 35 & $\begin{array}{l}\text { RSS Jwara. Chi., } \\
\text { sloka- } 42-43\end{array}$ & Vidyadhara rasa & $\begin{array}{l}\mathrm{Hg}, \mathrm{S}, \\
\text { TamraTankana }\end{array}$ & Trikatu,trivrith,vara,katuki & $\begin{array}{l}\text { Danthi, } \\
\text { dathura, } \\
\text { arka, } \\
\text { visha, } \\
\text { jaipala }\end{array}$ & $\begin{array}{l}\text { Snuhiksheera,dant } \\
\text { himoolakwatha }\end{array}$ \\
\hline 36 & $\begin{array}{l}\text { BR,Jwara chi. } \\
601-602\end{array}$ & Anjanabhairava rasa & $\begin{array}{l}\text { Sudha parada, } \\
\text { Tankana,Gandha } \\
\text { ka }\end{array}$ & Pippali & jayapala & Nimbuswarasa \\
\hline
\end{tabular}

\begin{tabular}{|c|c|c|c|c|c|c|}
\hline SL.No. & Text & Yoga & Minerals & Herbals & Visha/unavisha & Bhavana \\
\hline 1 & $\begin{array}{l}\text { RSS Jwara. } \\
\text { Chi., sloka-60 }\end{array}$ & Chandra sekhara rasa & $\begin{array}{l}\text { Suta,Gandhak } \\
\text { a, } \\
\text { Tankana,Man } \\
\text { asila }\end{array}$ & Maricha & & $\begin{array}{l}\text { Matsya } \\
\text { pitta }\end{array}$ \\
\hline 2 & $\begin{array}{lr}\text { RSS } & \text { Jwara. } \\
\text { Chi., } & \text { sloka- } \\
58,59 & \end{array}$ & Udaka manjari rasa & $\begin{array}{l}\text { Rasa,Gandhak } \\
\text { a,Tankana }\end{array}$ & Maricha,Sarkara & & $\begin{array}{l}\text { Meena } \\
\text { pitha }\end{array}$ \\
\hline 3 & $\begin{array}{l}\text { RSS Jwara. } \\
\text { Chi., sloka-61 }\end{array}$ & Pancha vaktra rasa & $\begin{array}{l}\text { Rasa,Gandhak } \\
\text { a,Naga,Tanka } \\
\text { na }\end{array}$ & Maricha,Pippali & & $\begin{array}{l}\text { Dhatura } \\
\text { swarasa }\end{array}$ \\
\hline 4 & $\begin{array}{lr}\text { RSS } & \text { Jwara. } \\
\text { Chi., } & \text { sloka- } \\
62-66 & \end{array}$ & $\begin{array}{l}\text { Parpata } \\
\text { rasa(parpatiprepare and } \\
\begin{array}{ll}\text { then bhavana with } \\
\text { nirgundi etc.then } \\
\text { swedana on angara) }\end{array}\end{array}$ & $\begin{array}{l}\text { Rasa,Gandhak } \\
\text { a,Tamra,Loha }\end{array}$ & & & $\begin{array}{l}\text { Bhringa } \\
\text { rasa,nirgun } \\
\text { di,Jayanthi,t } \\
\text { riphala,Kan } \\
\text { ya,vasa,Bha } \\
\text { rngi,Trikatu } \\
\text {,Chitraka,M } \\
\text { undi }\end{array}$ \\
\hline 5 & $\begin{array}{lr}\text { RSS } & \text { Jwara. } \\
\text { Chi., } & \text { sloka- } \\
67-69 & \end{array}$ & Vatha pithanthaka rasa & $\begin{array}{l}\text { Rasa,Abhra,T } \\
\text { amra,Theeksh } \\
\text { na,Loha,Maks } \\
\text { hika,Tala,Gan } \\
\text { thaka }\end{array}$ & Mustha & & $\begin{array}{l}\text { Yashti,Drak } \\
\text { sha,Amruth } \\
\text { a,dhathri,Sa } \\
\text { tavari,Kseer } \\
\text { a vidari }\end{array}$ \\
\hline 6 & $\begin{array}{lr}\text { RSS } & \text { Jwara. } \\
\text { Chi., } & \text { sloka- } \\
70-72 & \end{array}$ & Visweswara rasa & $\begin{array}{l}\text { Rasa,Tamra,T } \\
\text { heekshna,Tala } \\
\text { Gandhaka }\end{array}$ & & & \\
\hline 7 & $\begin{array}{lr}\text { RSS } & \text { Jwara. } \\
\text { Chi., } & \text { sloka- } \\
73-75 & \end{array}$ & Sithari rasa & $\begin{array}{l}\text { Rasa,Gandhak } \\
\text { a,Tankana }\end{array}$ & $\begin{array}{l}\text { Saindhava,maricha,chincha, } \\
\text { Twag,Bhasma,Sarkara }\end{array}$ & Jayapala & Jambi \\
\hline
\end{tabular}

Chart 5.3- Herbo mineral formulations in Sannipathajwara chikitsa

\begin{tabular}{|c|c|c|c|c|c|c|}
\hline Sl. No. & Reference & Yoga & Minerals & Herbals & Visha/upavisha & Bhavana \\
\hline 1 & $\begin{array}{l}\text { BR, Jwara. Chi., } \\
\text { sloka-591-593 }\end{array}$ & $\begin{array}{l}\text { Maha } \\
\text { jwarankusa rasa }\end{array}$ & Rasa gandhaka & Hemahwa & $\begin{array}{l}\text { Visha dhathura } \\
\text { bija }\end{array}$ & Jambiramajja \\
\hline 2 & $\begin{array}{l}\text { BR,Jwara } \\
\text { chi.594-595 }\end{array}$ & $\begin{array}{l}\text { Mohandandha } \\
\text { suryo } \\
\text { rasa(nasya) }\end{array}$ & Rasagandhaka & & & $\begin{array}{l}\text { Lasun } \\
\text { a rasa }\end{array}$ \\
\hline 3 & $\begin{array}{l}\text { RSS Jwara. } \\
\text { Chi., sloka-142- } \\
144\end{array}$ & $\begin{array}{l}\text { Brihath } \\
\text { badavanala rasa } \\
\text { (kupi pakva) }\end{array}$ & $\begin{array}{l}\text { Rasa gandhaka, } \\
\text { Tala,Daru, } \\
\text { sila,Abhraka }\end{array}$ & & $\begin{array}{l}\text { Jangama visha, } \\
\text { Visha, Jayapala }\end{array}$ & $\begin{array}{l}\text { Mathsya,Mahisha,May } \\
\text { ura,Chaga pitha }\end{array}$ \\
\hline 4 & $\begin{array}{lr}\text { RSS Jwara. } \\
\text { Chi., sloka-145- } \\
146\end{array}$ & $\begin{array}{l}\text { Suchikabhrana } \\
\text { rasa }\end{array}$ & Rasa,Gandhaka,Naga & & $\begin{array}{l}\text { Jangama } \\
\text { visha,Vatsanabha }\end{array}$ & $\begin{array}{l}\text { Matsya,Mayura,Chaga, } \\
\text { Varaha pitha }\end{array}$ \\
\hline 5 & $\begin{array}{l}\text { RSS Jwara. } \\
\text { Chi., sloka-147 }\end{array}$ & Panchanana rasa & $\begin{array}{l}\text { Gandhaka,Hingula,T } \\
\text { amra }\end{array}$ & Maricha & Vatsanabha & Arka,Ksheera \\
\hline 6 & $\begin{array}{l}\text { RSS Jwara. } \\
\text { Chi., sloka-148- } \\
149\end{array}$ & $\begin{array}{lr}\text { Tridosha } & \text { nihara } \\
\text { vinasaka } & \text { surya } \\
\text { rasa } & \end{array}$ & Rasa,gandhaka & & $\begin{array}{l}\text { Vatsanabha(1/8 of } \\
\text { rasa) }\end{array}$ & $\begin{array}{l}\text { Chitraka,Kwatha,Pitha } \\
\text { of } \\
\text { Matsya,Varaha,Chaga, } \\
\text { Mayura,Mahisha }\end{array}$ \\
\hline 7 & $\begin{array}{l}\text { RSS Jwara. } \\
\text { Chi., sloka-104- } \\
106\end{array}$ & $\begin{array}{l}\text { Ananda bhairava } \\
\text { rasa }\end{array}$ & Hingula, Tankana & $\begin{array}{l}\text { Vyosham, } \\
\text { Maricha, Kana, } \\
\text { Jatikosa }\end{array}$ & Visha & Jambira, Drava \\
\hline 8 & $\begin{array}{l}\text { RSS Jwara. } \\
\text { Chi., sloka-107- } \\
117\end{array}$ & $\begin{array}{l}\text { Ananda bhairavi } \\
\text { vatika }\end{array}$ & $\begin{array}{l}\text { Gandhaka, Tankana, } \\
\text { Tamra, Hingula }\end{array}$ & Trikatu & $\begin{array}{l}\text { Visha, Dhuthura } \\
\text { bija }\end{array}$ & Vijaya \\
\hline
\end{tabular}




\begin{tabular}{|c|c|c|c|c|c|c|}
\hline \multicolumn{7}{|c|}{ Chart 5.3 Continued... } \\
\hline 9 & $\begin{array}{l}\text { RSS Jwara. } \\
\text { Chi., sloka-131- } \\
132\end{array}$ & $\begin{array}{l}\text { Unmatta rasa for } \\
\text { nasya }\end{array}$ & Rasa, Gandhaka & & & Dhathura Phala \\
\hline 10 & $\begin{array}{l}\text { RSS Jwara. } \\
\text { Chi., sloka-133- } \\
138\end{array}$ & $\begin{array}{l}\text { Mritha, } \\
\text { Sanjeevana rasa } \\
\text { (kupi pakva) }\end{array}$ & Tamra, Tankana & & Visha, Jayapala & \\
\hline 11 & $\begin{array}{l}\text { RSS Jwara. } \\
\text { Chi., sloka-118- } \\
125\end{array}$ & $\begin{array}{l}\text { Praneswara rasa } \\
\text { ( kupi pakva) }\end{array}$ & Rasa, Gandhaka & & Visha & $\begin{array}{l}\text { Thalamuli, Rasa( } \\
\text { before kupi pakva), } \\
\text { Ajaji, Jeeraka, Hingu, } \\
\text { Sarjika, Yavakshara, } \\
\text { Yavanika, Maricha, } \\
\text { Pippali(kashaya of } \\
\text { them after preparation) }\end{array}$ \\
\hline 12 & $\begin{array}{l}\text { RSS Jwara. } \\
\text { Chi., sloka-139- } \\
141\end{array}$ & $\begin{array}{l}\text { Swalpa } \\
\text { badavanala rasa } \\
\text { (gajaputa) }\end{array}$ & Tamra & Maricha & Visha & Langali rasa \\
\hline 13 & $\begin{array}{lr}\text { RSS } & \text { Jwara. } \\
\text { Chi., } & \text { sloka-31- } \\
32 & \\
\end{array}$ & $\begin{array}{l}\text { Bhasmeswara } \\
\text { rasa }\end{array}$ & $\begin{array}{ll}\text { Aranya } & \text { upala } \\
\text { bhasma(16) } & \end{array}$ & Maricha(3) & Visha(1) & \\
\hline 14 & $\begin{array}{l}\text { RSS Jwara. } \\
\text { Chi., sloka-33 }\end{array}$ & $\begin{array}{l}\text { Chintamani } \\
\text { rasa(kupipakwa) }\end{array}$ & $\begin{array}{l}\text { Rasa,Gandhaka,Tank } \\
\text { ana,Tamra,Talaka. }\end{array}$ & $\begin{array}{l}\text { Yavakshara,Vyo } \\
\text { sha,Triphala }\end{array}$ & Visha & Madhu \\
\hline 15 & $\begin{array}{lr}\text { RSS } & \text { Jwara. } \\
\text { Chi., } & \text { sloka-86- } \\
87 & \\
\end{array}$ & $\begin{array}{l}\text { Kulavadhu rasa } \\
\text { (nasya)(kupipak } \\
\text { wa) }\end{array}$ & $\begin{array}{l}\text { Suta,Tamra,Naga,Ma } \\
\text { nassila,Thutha }\end{array}$ & & & Uthara varuni drava \\
\hline 16 & $\begin{array}{lr}\text { RSS } & \text { Jwara. } \\
\text { Chi., } & \text { sloka-88- } \\
90 & \end{array}$ & $\begin{array}{l}\text { Jayamangala } \\
\text { rasa(anjana,nasy } \\
\text { a)Bhudharaputa }\end{array}$ & $\begin{array}{l}\text { Rasa,Abhraka,Tara, } \\
\text { Munda,Theekshna,M } \\
\text { anasila,Makshika,Tan } \\
\text { kana }\end{array}$ & $\begin{array}{l}\text { Chitraka,Vyosha } \\
\text {,Pata,Nirgundi, } \\
\text { Vilwamoola }\end{array}$ & & \\
\hline 17 & $\begin{array}{l}\text { RSS Jwara. } \\
\text { Chi., sloka-91 }\end{array}$ & $\begin{array}{l}\text { Nasya bhairava } \\
\text { rasa }\end{array}$ & $\begin{array}{l}\text { Suta,Tamra,Theekshn } \\
\text { a ,Tankana,Kharpara }\end{array}$ & & & Arka dugdha \\
\hline 18 & $\begin{array}{l}\text { RSS Jwara. } \\
\text { Chi., sloka-92 }\end{array}$ & $\begin{array}{l}\text { Anjana bhairava } \\
\text { rasa }\end{array}$ & $\begin{array}{l}\text { Rasa,Theekshna } \\
\text { loha,Gandhaka }\end{array}$ & Kana & $\begin{array}{l}\text { Jayapala (3 times } \\
\text { of all) }\end{array}$ & Jambeera rasa \\
\hline 19 & $\begin{array}{l}\text { RSS Jwara. } \\
\text { Chi., sloka-93 } \\
\end{array}$ & Anjana rasa-1 & Rasa,Gandhaka & & & Lasuna rasa \\
\hline 20 & $\begin{array}{l}\text { RSS Jwara. } \\
\text { Chi., sloka-94 }\end{array}$ & Anjana rasa-2 & Rasa,Sulba,Tutha & Karpura,Hingu & & Kasamardarasa \\
\hline 21 & $\begin{array}{lr}\text { RSS } & \text { Jwara. } \\
\text { Chi., } & \text { sloka-95- } \\
98 & \end{array}$ & $\begin{array}{l}\text { Trilokya sundara } \\
\text { rasa }\end{array}$ & Rasa, Gandhaka & & & $\begin{array}{l}\text { Sakra,Musali,Dhattura, } \\
\text { Bhringa } \\
\text { raja,Devadali,jayanthi, } \\
\text { Manduka parni }\end{array}$ \\
\hline 22 & $\begin{array}{lr}\text { RSS } & \text { Jwara. } \\
\text { Chi., } & \text { sloka-99- } \\
102 & \\
\end{array}$ & $\begin{array}{l}\text { Swachanda } \\
\text { bhairava rasa }\end{array}$ & $\begin{array}{l}\text { Rasa,Gandhaka,Maks } \\
\text { hika }\end{array}$ & $\begin{array}{l}\text { Eeswari,Nagada } \\
\text {,Amalaki,Vandh } \\
\text { ya,Karkotaki }\end{array}$ & & \\
\hline 23 & $\begin{array}{l}\text { RSS Jwara. } \\
\text { Chi., sloka-183- } \\
185\end{array}$ & $\begin{array}{l}\text { Brihath kasthuri } \\
\text { bhairava rasa }\end{array}$ & $\begin{array}{l}\text { Tamra,Kanaka,Rajath } \\
\text { a,Muktha,Loha,vidru } \\
\text { ma,Kasthuri }\end{array}$ & $\begin{array}{l}\text { Karpura,Dhatha } \\
\text { ki,Suka,Simbi,P } \\
\text { ata,Vidanga,Mu } \\
\text { sta,Viswa,Usira, } \\
\text { Dhathri }\end{array}$ & & Arka dugdha \\
\hline 24 & $\begin{array}{l}\text { RSS Jwara. } \\
\text { Chi., sloka-189- } \\
191\end{array}$ & $\begin{array}{l}\text { Sannipata } \\
\text { badavanala rasa }\end{array}$ & $\begin{array}{l}\text { Rasa,Gandaka,Talaka } \\
\text {,tankana }\end{array}$ & Vyosha & Visha,Danthibija & Chitraka kwatha \\
\hline 25 & $\begin{array}{l}\text { RSS Jwara. } \\
\text { Chi., sloka-169- } \\
172\end{array}$ & Vethala rasa & $\begin{array}{l}\text { Rasa,Gandhaka,Mana } \\
\text { sila }\end{array}$ & Maricha & Visha & \\
\hline 26 & $\begin{array}{l}\text { RSS Jwara. } \\
\text { Chi., sloka-176 }\end{array}$ & $\begin{array}{l}\text { Kasthuri } \\
\text { bhairava rasa }\end{array}$ & Hingula, tankana & $\begin{array}{l}\text { Jatikosha } \\
\text { phala,maricha,Pi } \\
\text { ppali,Kasthuri }\end{array}$ & Visham & \\
\hline 27 & $\begin{array}{l}\text { RSS Jwara. } \\
\text { Chi., sloka-177- } \\
182\end{array}$ & $\begin{array}{l}\text { Brihath kasturi } \\
\text { bhairava rasa }\end{array}$ & $\begin{array}{l}\text { Vanga,Kharpara,Swa } \\
\text { rna,Thara,Kantha(4), } \\
\text { Makshika(2),Rasabha } \\
\text { sma }\end{array}$ & $\begin{array}{l}\text { Lavanga,Jathiko } \\
\text { saphala,Trikatu }\end{array}$ & & $\begin{array}{l}\text { Dronapushpi } \\
\text { rasa,Nagavalli rasa }\end{array}$ \\
\hline 28 & $\begin{array}{l}\text { RSS Jwara. } \\
\text { Chi., sloka-150- } \\
155\end{array}$ & $\begin{array}{l}\text { Rasarajendra } \\
\text { rasa(kupi } \\
\text { pakwa) }\end{array}$ & $\begin{array}{l}\text { Rasa,Tamra,Abhraka, } \\
\text { Ayas,Naga,Vanga,Ga } \\
\text { ndhaka,Thalaka }\end{array}$ & & Visha & $\begin{array}{l}\text { Kakamachi,Trikatu,Ar } \\
\text { draka,Rasa,Pitha(5) }\end{array}$ \\
\hline 29 & $\begin{array}{l}\text { RSS Jwara. Chi. } \\
\text { sloka -159-160 }\end{array}$ & $\begin{array}{l}\text { Mritha } \\
\text { sanjeevani rasa }\end{array}$ & $\begin{array}{l}\text { Suta,Gandha(2),Abhr } \\
\text { a,Loha,Tamra,Tala,V } \\
\text { aratika,Sila }\end{array}$ & Hingu & Visha & $\begin{array}{l}\text { Ardraka,Nirgundi,Vija } \\
\text { ya }\end{array}$ \\
\hline 30 & $\begin{array}{l}\text { RSS Jwara. } \\
\text { Chi., sloka-161- } \\
167\end{array}$ & $\begin{array}{l}\text { Gandhakakajjali } \\
\text { vidhi }\end{array}$ & Parade,Gandhaka & & & $\begin{array}{l}\text { Kantakari,Sindhuvari, } \\
\text { Nata,Karanja }\end{array}$ \\
\hline 31 & $\begin{array}{l}\text { RSS Jwara. } \\
\text { Chi., sloka-192- } \\
194\end{array}$ & Simha nada rasa & $\begin{array}{l}\text { Gandha,Rasa,Abhrak } \\
\text { a }\end{array}$ & & Vatsanabha & Barnigi drava,Nirgundi \\
\hline
\end{tabular}


Akhila Retnam. A et.al. A critical review of herbo-mineral formulations in jwara chikitsa.

\begin{tabular}{|c|c|c|c|c|c|c|}
\hline \multicolumn{7}{|c|}{ Chart 5.3 Continued... } \\
\hline 32 & $\begin{array}{l}\text { RSS Jwara. } \\
\text { Chi., sloka-195- } \\
198\end{array}$ & $\begin{array}{l}\text { Sannipatha surya } \\
\text { rasa }\end{array}$ & $\begin{array}{l}\text { Rasa,Gandhaka,Tamr } \\
\text { a,Tara hema }\end{array}$ & & Visha & $\begin{array}{l}\text { Chitrakakwatha,Matsy } \\
\text { adi pitha }\end{array}$ \\
\hline 33 & $\begin{array}{lr}\text { RSS } & \text { Jwara. } \\
\text { Chi., } & \text { sloka- } \\
199-203 & \end{array}$ & $\begin{array}{l}\text { Swachanda } \\
\text { nayaka rasa(kupi } \\
\text { pakwa) }\end{array}$ & $\begin{array}{l}\text { Suta,Gandhaka,Loha, } \\
\text { Tara }\end{array}$ & & & $\begin{array}{l}\text { Surya,Vartha,Nirgundi, } \\
\text { Tulasi,Girikarnika,Agn } \\
\text { ivalli,Ardrak,Vahni,Vij } \\
\text { aya,Jaya,Kakamachi,Pa } \\
\text { ncha pitha }\end{array}$ \\
\hline 34 & $\begin{array}{l}\text { RSS Jwara. } \\
\text { Chi., sloka-204- } \\
\text { 205 }\end{array}$ & $\begin{array}{l}\text { Sannipathanthak } \\
\text { a rasa }\end{array}$ & $\begin{array}{l}\text { Rasa,Gandha,Hingula } \\
\text {,Tamra,Kharpara }\end{array}$ & Amlavetasa & & Bhringaraja \\
\hline 35 & $\begin{array}{l}\text { BR,Jwara chi. } \\
827-830\end{array}$ & $\begin{array}{l}\text { Kasthuri } \\
\text { bhushana rasa }\end{array}$ & Rasa,Abhra,Tankana & $\begin{array}{l}\text { Sunti,Kasturi,Pi } \\
\text { ppali,Maricha,K } \\
\text { arpura }\end{array}$ & $\begin{array}{l}\text { Danthi mula,Jaya } \\
\text { bija }\end{array}$ & Ardraka rasa \\
\hline 36 & $\begin{array}{l}\text { BR, Jwara chi. } \\
831-832\end{array}$ & Arkamurthi rasa & $\begin{array}{l}\text { Loha(8),Tamra(1),Ra } \\
\text { sa(2),Gandhaka(4) }\end{array}$ & & Visha(1/16) & $\begin{array}{l}\text { Chithraka } \\
\text { kwatha,Pancha pitha }\end{array}$ \\
\hline 37 & $\begin{array}{l}\text { BR,Jwara chi. } \\
833-834\end{array}$ & $\begin{array}{l}\text { Tridosha } \\
\text { davanalo rasa }\end{array}$ & Arkamurthi rasa & & & $\begin{array}{l}\text { Nimba,Kantakari,Ardra } \\
\text { ka }\end{array}$ \\
\hline 38 & $\begin{array}{l}\text { RSS Jwara. } \\
\text { Chi., sloka-186- } \\
187\end{array}$ & Soubhagya vati & $\begin{array}{l}\text { Tankana ,lavana } \\
\text { panchaka,abhraka, } \\
\text { gandhaka }\end{array}$ & $\begin{array}{l}\text { Jeeraka } \\
\text { churna,vyosha,tr } \\
\text { iphala, } \\
\text { bhringaraja,vasa } \\
\text {, apamarga }\end{array}$ & Vathsanabhi & \\
\hline
\end{tabular}

Chart 5.4- List of Herbo mineral formulations for Jeerna and vishama jwara chikitsa

\begin{tabular}{|c|c|c|c|c|c|c|}
\hline SI no & Text & Yoga & Minerals & Herbals & $\begin{array}{l}\text { Visha / } \\
\text { upavisha }\end{array}$ & Bhavana \\
\hline 1 & 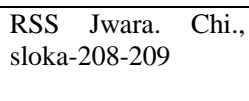 & $\begin{array}{l}\text { Jwarankusa } \\
\text { rasa }\end{array}$ & $\begin{array}{l}\text { Rasa,Gandhaka,Tankan } \\
\text { a }\end{array}$ & Maricha,Katphala & $\begin{array}{l}\text { Danthibij } \\
\text { a, } \\
\text { visha }\end{array}$ & \\
\hline 2 & $\begin{array}{ll}\text { RSS Jwara. } & \text { Chi., } \\
\text { sloka-210-214 } & \end{array}$ & $\begin{array}{l}\text { Jwarari } \\
\text { abhram }\end{array}$ & $\begin{array}{l}\text { Abhraka,Tamra,Rasa } \\
\text {,Gandhaka }\end{array}$ & Trikatu & $\begin{array}{l}\text { Visha, } \\
\text { Dhattura }\end{array}$ & Ardraka \\
\hline 3 & $\begin{array}{l}\text { RSS Jwara. } \\
\text { sloka-215-218 }\end{array}$ & Jwarasani rasa & $\begin{array}{l}\text { Rasa,Gandhaka,Saindha } \\
\text { va,Tamra,Loha,Abhraka }\end{array}$ & Maricha & Visha & Nirgundi \\
\hline 4 & $\begin{array}{l}\text { RSS Jwara. Chi., } \\
\text { sloka-219-221 }\end{array}$ & $\begin{array}{l}\text { Ardhanareesh } \\
\text { wara rasa }\end{array}$ & Rasa,Gandhaka & Maricha & $\begin{array}{l}\text { Jayapala, } \\
\text { visha }\end{array}$ & Triphala,jambhira \\
\hline 5 & $\begin{array}{l}\text { RSS Jwara. } \\
\text { sloka-222 }\end{array}$ & $\begin{array}{l}\text { Chandanadi } \\
\text { louha }\end{array}$ & Loha & $\begin{array}{l}\text { Raktachandana,Hribera,Patha, } \\
\text { Usira,Kana,Siva,nagara,Utpala } \\
\text {,Dathri,Trimada }\end{array}$ & & \\
\hline 6 & $\begin{array}{l}\text { RSS Jwara. } \\
\text { sloka-223-232 }\end{array}$ & Jwarari rasa & $\begin{array}{l}\text { Rasa,Gandhaka,Sila,Tal } \\
\text { a,Sulba }\end{array}$ & Trikatu & $\begin{array}{l}\text { Visha, } \\
\text { Dhathura }\end{array}$ & $\begin{array}{l}\text { Rohitaka } \\
\text { matsya,Pitha,Arka,A } \\
\text { rdraka }\end{array}$ \\
\hline 7 & $\begin{array}{ll}\text { RSS Jwara. } & \text { Chi., } \\
\text { sloka-233-239 } & \end{array}$ & $\begin{array}{l}\text { Sarvajwara } \\
\text { hara loha }\end{array}$ & Loha (equal to all) & Triphala,Trimada,Trikatu & & \\
\hline 8 & $\begin{array}{ll}\text { RSS Jwara. } & \text { Chi., } \\
\text { sloka-240-252 } & \end{array}$ & $\begin{array}{l}\text { Brihat Sarva } \\
\text { jwarahara loha }\end{array}$ & $\begin{array}{l}\text { Parada,Gandhaka,Tamra } \\
\text {,Abhraka }\end{array}$ & & & $\begin{array}{l}\text { Karavella,Dasamula, } \\
\text { Parpati }\end{array}$ \\
\hline 9 & $\begin{array}{l}\text { RSS Jwara. } \\
\text { sloka-253-260 }\end{array}$ & Maharaja vati & $\begin{array}{l}\text { Rasa,Gandhaka,Abhrak } \\
\text { a,Vanga,loha,Swarna,Ta } \\
\text { mra }\end{array}$ & $\begin{array}{lr}\text { Vridha } & \text { daru,Karpura, } \\
\text { Sakrasana } & \text { Vari,Lavanga, } \\
\text { Kokilaksha,Vidari,Musali,Suk } \\
\text { a,Jatiphala,Kosha,Bala,Nagaba } \\
\text { la }\end{array}$ & & Talamuli \\
\hline 10 & $\begin{array}{l}\text { RSS Jwara. } \\
\text { sloka-261-262 }\end{array}$ & $\begin{array}{l}\text { Chintamani } \\
\text { rasa(other) }\end{array}$ & $\begin{array}{l}\text { Swarna,Rajatha,Tala,M } \\
\text { ukta,Gandhaka,Parada }\end{array}$ & Trikatu,Kunati & & Kasthuri \\
\hline 11 & $\begin{array}{l}\text { RSS Jwara. } \\
\text { sloka-263-265 }\end{array}$ & $\begin{array}{l}\text { Trailokya } \\
\text { chintamani } \\
\text { rasa }\end{array}$ & $\begin{array}{l}\text { Swarna,Tara,Abhraka,L } \\
\text { oha,Pravala }\end{array}$ & & & Kanyarasa \\
\hline 12 & $\begin{array}{l}\text { RSS Jwara. } \\
\text { sloka-266-270 }\end{array}$ & $\begin{array}{l}\text { Brihath } \\
\text { chintamani } \\
\text { rasa }\end{array}$ & $\begin{array}{l}\text { Rasa,Gandhaka,Sila,Ro } \\
\text { upya,Tala,Swarna } \\
\text { tala,Mukta kasthuri }\end{array}$ & Trikatu,Triphala & Visha & $\begin{array}{l}\text { Bhringaraja,Tulasi,A } \\
\text { rdraka }\end{array}$ \\
\hline 13 & $\begin{array}{l}\text { RSS Jwara. } \\
\text { sloka-271-277 }\end{array}$ & $\begin{array}{l}\text { Putapakwa } \\
\text { vishama } \\
\text { jwaranthaka } \\
\text { loha }\end{array}$ & $\begin{array}{l}\text { Rasa Gandhaka,, } \\
\text { Swarna,Loha, Tamra, } \\
\text { Abhraka,Vanga,Mukta } \\
\text { sankh,Pravala }\end{array}$ & & & Ghritakumari \\
\hline 14 & $\begin{array}{l}\text { RSS Jwara. } \\
\text { sloka-278-284 }\end{array}$ & $\begin{array}{l}\text { Brihat } \\
\text { vishama } \\
\text { jwaranthaka } \\
\text { loha }\end{array}$ & $\begin{array}{l}\text { Rasa Gandhaka, } \\
\text { Rasasindura, Swarna, } \\
\text { Rajatha,Loha,Tamra, } \\
\text { Abhraka,Tala,Vanga,M } \\
\text { akshika,Mukta,Pravala }\end{array}$ & & & $\begin{array}{l}\text { Nirgundi,Nagavalli, } \\
\text { Kakamachi,Parpati,T } \\
\text { riphala,Karavella,Da } \\
\text { samla,Punarnava,Gu } \\
\text { duchi,Vasa,Bhringar } \\
\text { aja }\end{array}$ \\
\hline 15 & $\begin{array}{lcc}\text { RSS } & \text { Jwara. } & \text { Chi., } \\
\text { sloka-285-287 }\end{array}$ & $\begin{array}{l}\text { Seethabhanji } \\
\text { rasa(oter) } \\
\text { (baluka yantra } \\
\text { paka) }\end{array}$ & $\begin{array}{l}\text { Thalaka,parade, } \\
\text { gandhaka, sila, tamra }\end{array}$ & & & Naga valli\&maricha \\
\hline
\end{tabular}




\begin{tabular}{|c|c|c|c|c|c|c|}
\hline \multicolumn{7}{|c|}{ Chart 5.4 Continued... } \\
\hline 16 & $\begin{array}{l}\text { RSS Jwara. } \\
\text { sloka-288-289. }\end{array}$ & $\begin{array}{l}\text { Chinthamani } \\
\text { rasa } \\
\text { (other)(gajapu } \\
\text { ta) }\end{array}$ & $\begin{array}{l}\text { Thalaka,sulba,churna(su } \\
\text { kthi), thutha, }\end{array}$ & & & sarkara \\
\hline 17 & $\begin{array}{l}\text { RSS Jwara. } \\
\text { sloka-290-292 }\end{array}$ & $\begin{array}{l}\text { Jwarankusa } \\
\text { rasa(bhudhara } \\
\text { puta) }\end{array}$ & Tamra,Tala & & & Karavella,Vajripaya \\
\hline 18 & $\begin{array}{ll}\text { RSS Jwara. } & \text { Chi., } \\
\text { sloka-293-294 } & \\
\end{array}$ & $\begin{array}{l}\text { Meghanada } \\
\text { rasa (puta) }\end{array}$ & $\begin{array}{l}\text { Aara,Kamsya,Tamra,Ga } \\
\text { ndhaka }\end{array}$ & & & Meghanada \\
\hline 19 & $\begin{array}{l}\text { RSS Jwara. } \\
\text { sloka-295-296 }\end{array}$ & $\begin{array}{l}\text { Seetha } \\
\text { jwarahara rasa }\end{array}$ & $\begin{array}{l}\text { Makshika,Talaka,Rasa, } \\
\text { Gandhaka. }\end{array}$ & & $\begin{array}{l}\text { Bhallatak } \\
\text { a }\end{array}$ & Ravi snuhi \\
\hline 20 & $\begin{array}{l}\text { RSS Jwara. } \\
\text { sloka-297-300 }\end{array}$ & $\begin{array}{l}\text { Seethsbhanji } \\
\text { rasa }\end{array}$ & $\begin{array}{l}\text { Rasaka,Talaka,Tutha,Ta } \\
\text { nkana,Rasa,Gandhaka. }\end{array}$ & & & Karavella rasa \\
\hline 21 & $\begin{array}{l}\text { RSS Jwara. Chi., } \\
\text { sloka-301-305 }\end{array}$ & Panchana rasa & $\begin{array}{l}\text { Rasaka,Talaka,Tutha,Ta } \\
\text { nkana,Rasa,Gandhaka,T } \\
\text { amra. }\end{array}$ & & & Tulasi,Karavellaka \\
\hline 22 & $\begin{array}{l}\text { RSS Jwara. } \\
\text { sloka-306 }\end{array}$ & Vamana yoga & & Kumari moola kalka & & \\
\hline 23 & $\begin{array}{l}\text { RSS Jwara. } \\
\text { sloka-307-308 }\end{array}$ & $\begin{array}{l}\text { Visweswa } \\
\text { rasa }\end{array}$ & Darada gandhaka rasa & & & $\begin{array}{l}\text { Aswatha,Kola,Nigad } \\
\text { hika,Kakamachi. }\end{array}$ \\
\hline 24 & $\begin{array}{l}\text { RSS Jwara. } \\
\text { sloka-309-310 }\end{array}$ & $\begin{array}{l}\text { Tryahikari } \\
\text { rasa }\end{array}$ & Rasaka,Sanka Tutha & & & $\begin{array}{l}\text { Go jihwa,Jayanthi, } \\
\text { Tanduleeyaka }\end{array}$ \\
\hline 25 & $\begin{array}{l}\text { RSS Jwara. Chi., } \\
\text { sloka- } 311-313\end{array}$ & $\begin{array}{l}\text { Chaturthakari } \\
\text { rasa }\end{array}$ & $\begin{array}{l}\text { Tala } \\
\text { sila,Tutha,Gandhaka,Sa } \\
\text { nkha }\end{array}$ & & & Kumari \\
\hline 26 & $\begin{array}{l}\text { RSS Jwara. Chi., } \\
\text { sloka-314-316 }\end{array}$ & $\begin{array}{l}\text { Chinthamani } \\
\text { rasa }\end{array}$ & $\begin{array}{l}\text { Rasa,Gandhaka,Sulba,A } \\
\text { bhra }\end{array}$ & Trikatu,Triphala & $\begin{array}{l}\text { Danthi } \\
\text { visha }\end{array}$ & Drona pushpin rasa \\
\hline 27 & $\begin{array}{l}\text { RSS Jwara. Chi., } \\
\text { sloka-317-322 }\end{array}$ & $\begin{array}{l}\text { Brihath } \\
\text { chinthamani } \\
\text { rasa }\end{array}$ & $\begin{array}{l}\text { Rasa Gandhaka,Swarna, } \\
\text { Rajatha,Loha,Tamra,Tal } \\
\text { a,Kharpara,Kamsya,Van } \\
\text { ga,Makshika,Kaseesa,Si } \\
\text { la,Tankana,Mukta,Vidru } \\
\text { ma }\end{array}$ & Karpura & & $\begin{array}{l}\text { Bharngi,Vasa,Nirgun } \\
\text { di,Nagavalli,Jayanthi } \\
\text {,Karavella,Patola,Bh } \\
\text { anga, Punarnava } \\
\text {,Ardraka }\end{array}$ \\
\hline 28 & $\begin{array}{l}\text { RSS Jwara. } \\
\text { sloka-323-328 }\end{array}$ & $\begin{array}{l}\text { Maha } \\
\text { jwarankusa } \\
\text { rasa }\end{array}$ & $\begin{array}{l}\text { Rasa Gandhaka,Loha, } \\
\text { Tamra,Abhraka,Tala,Va } \\
\text { nga,Makshika,Kharpara, } \\
\text { Manasila, } \\
\text { Abhraka,gairika, } \\
\text { Tankana,hingula }\end{array}$ & & Danthi & $\begin{array}{l}\text { Jambira,Vijaya,Chitr } \\
\text { aka.Tulasi,Tinthidika }\end{array}$ \\
\hline 29 & $\begin{array}{l}\text { RSS Jwara. } \\
\text { sloka-331-339 }\end{array}$ & $\begin{array}{l}\text { Sarvatho } \\
\text { bhadra rasa }\end{array}$ & $\begin{array}{l}\text { Abhraka,gandhaka,para } \\
\text { da }\end{array}$ & $\begin{array}{l}\text { Kesara,karpura,mamsi, teja } \\
\text { patra,jathi kosha,sukshmaila, } \\
\text { pippali, kushta, thalisa pathra, } \\
\text { harithaki, dhathaki etc. }\end{array}$ & & $\begin{array}{l}\text { Madhu, sitha, parna } \\
\text { khanda }\end{array}$ \\
\hline 30 & $\begin{array}{l}\text { BR,Jwara. Chi., } \\
\text { sloka-1077-1079 }\end{array}$ & $\begin{array}{l}\text { Vidya } \\
\text { vallabho rasa }\end{array}$ & Rasa,Tamra,Sila,Talaka & & & Karavellaka \\
\hline 31 & $\begin{array}{l}\text { RSS Jwara. Chi., } \\
\text { sloka-173-175 }\end{array}$ & $\begin{array}{l}\text { Chandra } \\
\text { sekhara rasa }\end{array}$ & $\begin{array}{l}\text { Rasa, gandhaka, } \\
\text { tankana,manasila }\end{array}$ & & & Mathsya pitta \\
\hline
\end{tabular}

\section{DISCUSSION}

Rasasastra is a unique technique for converting poisonous metal, mineral, and herbal raw drugs into safer pharmaceutical preparations for the wellbeing of humanity. In comparison to conventional herbal formulations, herbo mineral formulations are more stable and interactive, resulting in faster therapeutic effect and a longer shelf life. It is well known that they offer significant therapeutic promise in the treatment of all diseases. The majority are rasayanas and yogavahis. They are extremely powerful for aamadosha nirharana. Because of their fine particle size, they have the ability to act in a subtle level. Nowadays, nano particles are used to deliver drugs to specific cell types ${ }^{20}$. Particles are designed to attract diseased cells, allowing direct treatment of specific cells while minimizing damage to healthy cells $^{21}$.

According to Ayurveda, Pitha regulates body temperature. Pitha pradhana dosa dushti \& ama dosha are the main causes of jwara. As a result, pitha hara, srotosodhaka, deepana pachana, and sweda janana qualities should be present in jwaraghna dravyas. The most prevalent herbal constituents in these compositions include sunti, pippali, maricha, kumari, and triphala. They have all of the jwaraghna 
qualities. On the other hand, kajjali, rasa sindura, parada, hingula, and other rasoushadhies act as bio enhancers to effectively augment the qualities of the above medications. Rasaushadies also have sadyo deepana pachana qualities and are particularly effective in ama dosha nirharana.

"Fever is defined as the elevation of body temperature above $37^{0} \mathrm{C}$. This occurs as a result of hypothalamic "set point" for temperature. Once hypothalamic set point is raised neurons in the vasomotor centre are stimulated leading to peripheral vasoconstriction especially in the limbs, leading to decreasing the heat loss from the skin. Metabolic processes also being slow and the body temperature elevates gradually and steadily" 22 . The antipyretic drugs act centrally, resetting the mechanism and bringing the temperature back to normal. Antipyretic medicines work by preventing Prostaglandin production and release in the brain $^{23}$. Increased heat dissipation is mostly caused by cutaneous vasodilatation, which is accompanied by perspiration, which aids in the decrease of body temperature ${ }^{24}$. Studies reveal that Ayurveda medicines which have antipyretic property also reduce the level of prostaglandins in body ${ }^{25}$.

There are some peculiarities among the yogas mentioned for jwara chikithsa in rasasastra classics. On considering nava jwara, they are mostly kharaleeya rasayanas. Because of their popularity, wide range of therapeutic usefulness, ease of preparation, and mode of administration, Kharaleeya rasayanas take the lead over the other three rasayanas, parpati, pottali, and kupipakwa. By the act of triturating, the entire phase of these preparations takes place in khalva yanthra, hence the name kharaleeya or khalveeya rasayanas. Triturating not only reduces particle size to a very fine level, but it also aids in the breakage and reunion of bonds between triturated particles. Hence, the finished product is a completely different compound in composition ${ }^{26}$.
Mineral ingredients present in majority of the yogas include Parada, Gandhaka, Hingula, and Tankana. To begin, sodhitha parada and Gandhaka are properly triturated and kajjali is prepared. Then check for colour, varithrathwa, rekhapoornathwa, as well as other sidhalakshanas of the kajjali. The remaining herbal and mineral ingredients, as well as the kajjali and appropriate bhavana dravyas specified in yoga, are triturated later ${ }^{27}$.

Kajjali (black sulphide of mercury) is a one-of-a-kind remedy that can be used to treat a wide range of ailments when combined with the appropriate anupana and sahapana. Sarvamayahara, vrishya, and tridoshahara are the properties of this preparation ${ }^{28}$. Kajjali works synergistically and comprehensively with the herbal ingredients to provide a multi-targeted organ effect. In clinical practice, drugs containing kajjali have a higher rate of timed and sustained release than drugs without kajjali. Vyosha, maricha, nagara, and pippali are herbal ingredients with agni deepana and kapha- vata samana properties. Other ingredients include katuki, thrivruth, harithaki, vasa, and nimba. They have kapha pitta samana and virechana effects. Tiktha and Katu rasas are present in majority of these medicines. Katu rasa has deepana and ushna veerya, while Tiktha rasa has jwarahara quality. Deepana, Pacana, Lekhana, and Krimighna properties are present in majority of these medicines. Sunti is present in most formulations, and its excellent ama pachana property aids in the samprapti vighatana of jwara. The presence of visha and upavishadravyas is another unique feature of these yogas. The main ingredients in these formulations are vatsanabhi, danthi, jayapala, and arka. Their concentration is $2 / 4 / 8$ times that of other ingredients in some of the formulations. In certain yogas, they are used as bhavana dravyas.

According to Rasa Tarngini, vathsanabha is a sreshta rasayana when paired with rasa. Agnideepana, vatha kapha samana, seetha samana, and jwarahara are 
the properties of Vathsanabhi ${ }^{29}$. According to Ayurveda Prakasa, the toxic effects of the compound will be totally removed if the vathsanabhi is combined with an equivalent amount of properly sodhitha tankana.

Purified Jayapala has a powerful purgative effect and is useful in both jalodara and navajwara ${ }^{30}$. Sodhitha dathura beeja has krimighna and kushta samana properties and is used primarily in jwara $^{31}$. In jwara, arka ksheera is purgative and helpful for reducing srothorodha ${ }^{32}$. Madhu, sringaverambu, and ardraka swarasa are recommended as anupana in almost all yogas. Specific anupanas are recommended for different jwaravasthas in some yogas. Dose of these formulations usually range from 1 ratti (125mg) to 1 valla $(750 \mathrm{mg})$. A detailed examination of the yogas mentioned in nava jwara reveals that they are all excellent combinations of drugs with antipyretic, antibacterial, and antiviral properties. Most of the formulations in nirama jwara include Parada and Gandhaka in Kajjali form. Other mineral ingredients include tankana, abhraka, tamra, and loha. Except in Sithari rasa, the visha and upavisha groups of drugs are not included in these formulations. Different anupanas mentioned depending on the dosha of the jwara, for example, pathya sunti amrutha kwatha and thaptha thoya in vatha- kapha jwara, ksheera and sitha in paithika jwara, and madhu, kakamachi, and saindhava in kapha jwara. In Rasa Rathna Samucchaya, Vagbhata does not mention visha kalpas for nirama jwara.

Almost all metallic and mineral bhasmas are used in sannipatha jwara hara yogas such as Rasa, Gandhaka, Manasila, Thalaka, Hingula, Tamra, Loha, Makshika, Naga, Vanga. Vathsnabha, upavishas such as dhathura, arka, jaipala, bhallathaka, danthi, jangama visha, such as sarpavisha, and toxic minerals such as manasila, harithala, and gouri pashana are used in almost all formulations. Since the ingredients in sannipatha jwara yogas are highly toxic, the majority of these yogas are formulated as kupi pakva rasayanas, which are much safer due to their special preparation technique, or prepared with bhudhara puta or gaja puta ${ }^{33}$. Herbs used for bhavana include nirgunti, thulasi, girikarnika, agni valli, ardraka, vahni, vijaya, jaya, kakamachi, drona pushpi, nagavalli, bhringa raja, trikatu, and thalamuli. For bhavana, pancha pithas, (mathsya, barhi, varaha, mahisha, and gavya ${ }^{34}$ ) are also used. Pancha pithas (pithas obtained from many animal sources) are utilised for bhavana in sannipatha jwara yogas. According to Rasendra Sara sangraha by using such medicines, one should follow jala seka in moordha, jala parisheka avagaha, such as cold therapy regimens, which helps to balance the theekshnatha of medicine in the body ${ }^{35}$. Krishna sarpa visha is very theekshna and ushna in nature. So it is indicated in seethanga sannipatha, seethe gathratha (coldness of body in sannipatha jwara) by RasaTarangini. Along with karaskara, and kasthuri it is effective in various sannipatha avasthas like sanja nasa (unconsciousness), swasa krichratha (dyspnoea), indriya saithilya( unable to perceive sensations). This combination is found in Kasthuri bhairava rasa, and Brihath kasthuri bhairava rasa ${ }^{36}$. However the Krishna sarpa visha is not advised for jeerna jwara, sanyasa, other jeerna vikaras and rakthapitha ${ }^{37}$.

These formulations even have kasthuri and karpura in them. Plenty of yogas are used for nasya and anjana in sannipatha avastha. Theekshna and ushna are the characters in the bhavana dravyas used for applying nasya and anjana formulations. Nasya karma is performed with preparations such as Nasya bhairava, Jayamangala rasa, Kula vadhu rasa, Mohandha surya rasa, and Unmatta rasa. Anjana karma is performed using Anjana bhairava rasa $1 \& 2$ and Jaya mangala rasa. Eyes and nostrils are being the convenient route of drug administration in unconscious patients. So, in Sanniatha avastha, medicines are administered as Nasya and 
Anjana, they function directly on the CNS. Pippali, maricha, vacha, and herbs with theekshna properties are also used for anjana and nasya. The way Suchikabharana rasa is administered is also unique. It can be applied to the scalp, the brahma randhra, or the talu. Otherwise, for the administration of Suchikabharana rasa, a slight needle prick or scratch would be made in the brahma randhra or talu pradesa. Nalikerodaka, vyosha, ardraka swarasa are commonly used anupanas in sannipatha jwara.

Mineral ingredients used in jeerna and vishama jwaraavastha include loha, tamra, gandhaka, swarna, abhra, and rajatha. Muktha, muktha sukthi, pravala, and sanka are special calcium carbonatebased ingredients used in these yogas. These drugs have vahnimandya hara, deepani, pleeha roga hara properties which are the main lakshanas of jeerna jwra ${ }^{38}$. The key herbal ingredients are raktha chandana, hreebera, pata, usira, kana, siva, nagara, uthpala, dhathri, maricha, trikatu, and triphala. Two abhra yogas and many loha kalpas are specially mentioned for jeerna and vishama jwara chikithsa. Chandanadi louha, vishama jwaranhaka louha, sarva jwaranthaka louha etc. are some of the most popular louha kalpas.

On analyzing the yogas, it is clear that much care was given in selection of drugs, preparation and administration of them (bhavana and anupana) to achieve maximum potency for each condition. These mineral-based compositions are ideal for the corresponding jwara avsthas. Similarly, the mode of administration and method of preparation are particularly adaptable to a variety of scenarios. Medicines for nava jwara avastha, for example, are easier to make and administer. They're mostly kharaleeya rasayanas. Whereas in sannipatha jwara, the majority of the yogas are kupipakva or puta preparations that can be delivered by a variety of routes, including nasya, anjana, sublingual, scalp, and so on. These yogas are finer than kharaleeya rasayanas since they are made by sublimation of ingredients. We may conclude that Rasasastra is a unique methodology for the bimolecular remodeling of metal, mineral, and herbal medicines for the benefit of humanity.

\section{CONCLUSION}

Things being so, the herbal ingredients in combination with the appropriate Rasaoushadhis have the ability to effectively treat various jwara avasthas. An examination of these textbooks revealed that they contain a wealth of herbo-mineral formulations capable of treating from minor symptoms to severe complications of jwara such as vathika navajwara avastha to complex sannipatha conditions. Since they are not packaged and marketed by pharmaceutical companies, many of them are unfamiliar with standard practice. As a result, Rasasatra's absolute potency is unable to address society's fever issues effectively. Therefore, our health-care system must adopt these medications which meet all of the requirements of a superior medicine for the community's wellbeing.

\section{Acknowledgement: None}

\section{Conflict of Interest: None}

\section{Source of Funding: None}

Ethical Approval: Not required

\section{REFERENCES}

1. Anuja Susan Varghese, The New Indian Express, "Fever kills scores in Kerala every year, but such deaths go unreported" Published: $30^{\text {th }}$ July 2020, Reported at Kochi.

2. Prof. Priyavratha Sarma, Editor and Translator, Charaka samhitha vol.II, Chikithsa sthana, Chaukhambha orientalia, Varanasi,Edition- $7^{\text {th }}$, Chapter- 3, Page 53,Sloka -11

3. Prof. Priyavratha Sarma, Editor and Translator, Charaka samhitha vol.II, Chikithsa sthana, Chaukhambha orientalia, Varanasi, Edition- $7^{\text {th }}$, Chapter- 3, Page 53,Sloka -12

4. Prof. Priyavratha Sarma Editor and Translator, Charaka samhitha vol.II, 
Chikithsa sthana, Chaukhambha orientalia, Varanasi, Edition- $7^{\text {th, }}$, Chapter- 3, page 54,sloka -26

5. Dr. Kanjiv Lochan., Bhaishajya Ratnavali of Sri Govind Dasji , English translation vol-1, Published by Chaukhambha Sanskrit Bhawan, Post Box No. 1160, Varanasi221001(India), Edition -first,2006, Chapter5, Page-97, Sloka-12

6. Sri. P. M. Govindan Vaidyn's Malayalam Translation of Ashtanga hridaya , Nidana Sthana, Published by Devi book stall, Sringa puram, Kodungalloor- 680664, Chapter-2, Sloka $-3,4,5$

7. Prof. Priyavratha Sarma, Editor and Translator, charaka samhitha vol.II, Chikithsa sthana, Chaukhambha orientalia, Varanasi, Edition- $7^{\text {th, }}$,Chapter- 3, Page -55, Sloka $-32-35$

8. Prof. Priyavratha Sarma Editor and Translator, C-haraka samhitha vol.II, Chikithsa sthana, Chaukhambha orientalia, Varanasi, Edition- $7^{\text {th }}$, Chapter- 3, Page 57,Sloka -51

9. Dr. Kanjiv Lochan., Bhaishajya Ratnavali of Sri Govind Dasji , English translation vol-1, Published by Chaukhambha Sanskrit Bhawan, Post Box No. 1160, Varanasi221001(India), Edition -first, 2006, Chapter-5, Page-95, Sloka-5

10. Dr. Kanjiv Lochan., Bhaishajya Ratnavali of Sri Govind Dasji , English translation vol-1, Published by Chaukhambha Sanskrit Bhawan, Post Box No. 1160, Varanasi221001(India), Edition -first, 2006, Chapter-5, Page-95, Sloka-6

11. Dr. Kanjiv Lochan., Bhaishajya Ratnavali of Sri Govind Dasji , English translation vol-1, Published by Chaukhambha Sanskrit Bhawan, Post Box No. 1160, Varanasi221001 (India), Edition -first, 2006, Chapter-5, Page-104, Sloka-43

12. Dr. Kanjiv Lochan, Bhaishajya Ratnavali of Sri Govind Dasji, English translation vol-1, Published by Chaukhambha Sanskrit Bhawan, Post Box No. 1160, Varanasi221001(India),Edition -first,2006, Chapter5, page-399, sloka-1434

13. Prof. Priyavratha Sarma, Editor and Translator, Charaka samhitha vol.II, Chikithsa sthana, Chaukhambha orientalia, Varanasi, Edition- ${ }^{\text {th }}$, Chapter- 3

14. Dr. Kanjiv Lochan., Bhaishajya Ratnavali of Sri Govind Dasji , English translation vol-1, Published by Chaukhambha Sanskrit
Bhawan, Post Box No. 1160, Varanasi221001(India),Edition -first,2006, Chapter5, page-160,sloka-213

15. Dr. Kanjiv Lochan., Bhaishajya Ratnavali of Sri Govind Dasji, English translation vol-1, Published by Chaukhambha Sanskrit Bhawan, Post Box No. 1160, Varanasi221001(India),Edition -first,2006, Chapter5, page-160,sloka-214

16. Prof. Priyavratha Sarma Editor and Translator, charaka samhitha vol.II, Chikithsa sthana, Chaukhambha orientalia, Varanasi,Edition- $7^{\text {th, }}$ Chapter- 3, page 59 ,sloka -73

17. Dr. Kanjiv Lochan, Bhaishajya Ratnavali of Sri Govind Dasji , English translation vol-1, Chapter-5, Published by Chaukhambha Sanskrit Bhawan, Post Box No. 1160, Varanasi-221001(India),Edition -first,2006, page-104,sloka-45

18. Dr. Kanjiv Lochan., Bhaishajya Ratnavali of Sri Govind Dasji , English translation vol-1, Published by Chaukhambha Sanskrit Bhawan, Post Box No. 1160, Varanasi221001(India),Edition -first,2006, Chapter5, sloka-1393

19. Dr. Parimi Suresh, Rasendra Sara Sangraha of Sri Gopala Krishna Bhatt, English Translation, Published by Chaukhambha Sanskrit Sansthan,post box no.1139,K37/116, Gopal Mandir Lane, Varansi-221001(India), Edition: First, 2007, Chapter-2, p- 110 0nwards

20. Vanna Sanna, Nicolino Pala, and Mario Sechi, Targeted therapy using nanotechnology: focus on cancer, International Journal of Nano medicine, ncbi.nlm, nih.gov

21. Vanna Sanna, Nicolino Pala, and Mario Sechi, Targeted therapy using nanotechnology: focus on cancer, International Journal of Nano medicine, ncbi.nlm, nih.gov

22. K. V KrishnaDas, Textbook of Medicine,Published by Jitendar P Vij, Jaypee Brothers publishers(P) Ltd, $5^{\text {th }}$ Edition,2008,Chapter-34-Diseases caused by infections, p- 176

23. K. V KrishnaDas, Textbook of Medicine,Published by Jitendar P Vij, Jaypee Brothers publishers $(\mathrm{P})$ Ltd, $5^{\text {th }}$ Edition,2008,Chapter-34-Diseases caused by infections, p- 177

24. K. V KrishnaDas, Textbook of Medicine,Published by Jitendar P Vij, 
Jaypee Brothers publishers(p) LTD， $5^{\text {th }}$ Edition,2008,Chapter-34-Diseases caused by infections, p- 176

25. Manoj J. Timbadia, K. Nishteswar and Mukesh B. Nariya, "Experimental evaluation of antipyretic and analgesic activities of Amalakyadi Gana: An Ayurvedic Formulation", Wolters KluwerMedknow Publications, AYU, ncbi.nlm.nih.gov

26. Dr.Ravindra Angadi, A Textbook of Rasasastra, Published by Chaukhambha Surbharati Prakashan, Varanasi, Chapter 13, Page-140

27. Dr.Ravindra Angadi, A Textbook of Rasasastra, Published by Chaukhambha Surbharati Prakashan, Varanasi, Chapter 13, Page-141

28. Pandit Kasinatha Sasthri, Rasa Tarngini of Pranacharya Sadananda Sarma,published by Mothilal Banarasi Das, 41 U M. Banglow road, Jawahar Nagar, New Delhi, Murchanadi vijnaneeya $6^{\text {th }}$ Tharanga, sloka no. 112,page no. 126

29. Pandit Kasinatha Sasthri, Rasa Tarngini of Pranacharya Sadananda Sarma,published by Mothilal Banarasi Das, 41 U M. Banglow road, Jawahar Nagar, New Delhi, 24 Tharanga, sloka no. 26-27,page no. 653

30. Pandit Kasinatha Sasthri, Rasa Tarngini of Pranacharya Sadananda Sarma,published by Mothilal Banarasi Das, 41 U M. Banglow road, Jawahar Nagar, New Delhi, Vishopavisha vijnaneeya $24^{\text {th }}$ Tharanga, sloka no. 315-316, page no. 706

31. Pandit Kasinatha Sasthri, Rasa Tarngini of Pranacharya Sadananda Sarma,published by Mothilal Banarasi Das, 41 U M. Banglow road, Jawahar Nagar, New Delhi, Vishopavisha vijnaneeya $24^{\text {th }}$ Tharanga, sloka no. 350-351, page no. 711

32. Pandit Kasinatha Sasthri, Rasa Tarngini of Pranacharya Sadananda Sarma,published by Mothilal Banarasi Das, 41 U M. Banglow road, Jawahar Nagar, New Delhi,
Vishopavisha vijnaneeya $24^{\text {th }}$ Tharanga, sloka no. 511-512, page no. 743

33. Dr. Parimi Suresh, Rasendra Sara Sangraha of Sri Gopala Krishna Bhatt, English Translation, Published by Chaukhambha Sanskrit Sansthan,post box no.1139,K37/116, Gopal Mandir Lane, Varansi-221001(India), Edition: First, 2007, Chapter-2, p- 110 0nwards

34. Dr. Parimi Suresh, Rasendra Sara Sangraha of Sri Gopala Krishna Bhatt, English Translation, Published by Chaukhambha Sanskrit Sansthan,post box no.1139,K37/116, Gopal Mandir Lane, Varansi-221001(India), Edition: First, 2007, Chapter-2, sloka- 168 p-157

35. Dr. Parimi Suresh, Rasendra Sara Sangraha of Sri Gopala Krishna Bhatt, English Translation, Published by Chaukhambha Sanskrit Sansthan,post box no.1139,K37/116, Gopal Mandir Lane, Varansi-221001(India), Edition: First, 2007, Chapter-2, sloka- 168,p-156

36. Pandit Kasinatha Sasthri, Rasa Tarngini of Pranacharya Sadananda Sarma,published by Mothilal Banarasi Das, 41 U M. Banglow road, Jawahar Nagar, New Delhi, Vishopavisha vijnaneeya $24^{\text {th }}$ Tharanga, sloka no. 538-546, page no. 748

37. Pandit Kasinatha Sasthri, Rasa Tarngini of Pranacharya Sadananda Sarma,published by Mothilal Banarasi Das, 41 U M. Banglow road, Jawahar Nagar, New Delhi, Vishopavisha vijnaneeya $24^{\text {th }}$ Tharanga, sloka no. 547-548, page no. 749

38. Pandit Kasinatha Sasthri, Rasa Tarngini of Pranacharya Sadananda Sarma,published by Mothilal Banarasi Das, 41 U M. Banglow road, Jawahar Nagar, New Delhi, Sankadi vijnaneeya $12^{\text {th }}$ Tharanga, page no. 285-306

How to cite this article: Akhila Retnam. A, Keerthi V. R, Mathew R et.al. A critical review of herbo-mineral formulations in jwara chikitsa. Int J Health Sci Res. 2021; 11(7): 394-406. DOI: https://doi.org/10.52403/ijhsr.20210754 